\title{
Estrogen-related receptor $\alpha$ is involved in the osteogenic differentiation of mesenchymal stem cells isolated from human periodontal ligaments
}

\author{
CHUAN CAI ${ }^{1}$, GONG-JIE YUAN ${ }^{1,2}$, YE HUANG $^{3,4}$, NAN YANG $^{1,5}$, XIN CHEN $^{1,6}$, \\ LI WEN $^{1}$, XI WANG $^{1}$, LIANG ZHANG $^{1}$ and YIN DING ${ }^{1}$
}

\begin{abstract}
${ }^{1}$ Department of Orthodontics, School of Stomatology, The Fourth Military Medical University, Xi'an 710032;
${ }^{2}$ Department of Orthodontics, Dalian Stomatological Hospital, Dalian 116002; ${ }^{3}$ Affiliated Hospital of Institute of Aviation Medicine, Air Force, Beijing 100089; ${ }^{4}$ Department of Dermatology, General Hospital of Air Force, Beijing 100142; ${ }^{5}$ Department of Stomatology, The PLA 309th Hospital, Beijing 100091; ${ }^{6}$ Department of General Dentistry, The 174th Hospital of Chinese PLA, Xiamen 361003, P.R. China
\end{abstract}

Received December 18, 2012; Accepted February 25, 2013

DOI: $10.3892 /$ ijmm.2013.1305

\begin{abstract}
Recently, it has been reported that the orphan nuclear receptor estrogen-related receptor $\alpha(E R R \alpha)$ is involved in the osteogenic differentiation of mesenchymal stem cells (MSCs). Moreover, ERR $\alpha$ has been identified as a novel therapeutic target for treating osteoporosis and other bone diseases. Human periodontal ligament tissue-derived mesenchymal stem cells (hPDLSCs) have recently been used in stem cell-mediated therapies because of their multipotency, particularly toward osteogenic differentiation. However, it is still unclear whether ERR $\alpha$ can regulate the osteogenic differentiation of hPDLSCs. In the present study, we investigated the role of ERR $\alpha$ in the osteogenic differentiation of hPDLSCs in vitro. We isolated hPDLSCs and confirmed their capacity for multipotent differentiation. Furthermore, we examined ERR $\alpha$ expression in hPDLSCs by RT-PCR and immunocytochemistry. We found that the expression of ERR $\alpha$ mRNA was significantly increased during the late stage of osteogenic differentiation of hPDLSCs. Moreover, transfection of recombinant lentiviral-mediated miRNA targeting ERR $\alpha$ significantly suppressed ALP activity, mineralization capacity, and the mRNA expression of osteogenesis-related genes (ALP, OCN, RUNX2 and OPN) in hPDLSCs. Our results indicate that ERR $\alpha$ may promote the osteogenic differentiation of hPDLSCs in vitro.
\end{abstract}

\section{Introduction}

Estrogen-related receptor $\alpha(E R R \alpha, N R 3 B 1)$ is among the first orphan nuclear receptors identified by low-stringency

Correspondence to: Professor Yin Ding, Department of Orthodontics, School of Stomatology, The Fourth Military Medical University, 145 West Changle Road, Xi'an 710032, P.R. China

E-mail: yin.ding@hotmail.com

Key words: estrogen-related receptor $\alpha$, osteogenic differentiation, mesenchymal stem cells, periodontal ligament screening of cDNA libraries with a probe encompassing the DNA binding domain of human estrogen receptor (ER) $\alpha$ (1). ERR $\alpha$ shares only $37 \%$ amino acid identity in its ligand binding domain with $\mathrm{ER} \alpha(1)$, which may explain the fact that ERR $\alpha$ does not bind estrogen. Instead, ERR $\alpha$ activates gene transcription constitutively in a ligand-independent manner (2). ERR $\alpha$ is involved in various physiological regulatory processes. It is a regulator of energy metabolism $(3,4)$, and is essential for adaptive thermogenesis (5). ERR $\alpha$ is also related to the growth and progression of several gynecological cancers $(6,7)$. ERR $\alpha$ is regulated by estrogen in bone, and it may play a functional role in diseases caused by estrogen deficiency, such as osteoporosis (8). ERR $\alpha$ is expressed throughout osteoblastic differentiation and may regulate bone formation both in vitro (9) and in vivo (10), and it is also involved in osteoclast adhesion and transmigration (11). Since osteoblasts arise from multipotent mesenchymal stem cells (MSCs), several studies have investigated the role of ERR $\alpha$ in the osteogenic differentiation of MSCs. Various studies have designated ERR $\alpha$ as an activator of osteogenic differentiation of MSCs $(12,13)$, whereas other studies have suggested that ERR $\alpha$ is an inhibitor $(14,15)$. Therefore, the function of ERR $\alpha$ in osteogenic differentiation of MSCs has not been clearly understood.

Human periodontal ligament tissue-derived mesenchymal stem cells (hPDLSCs), first isolated by Seo et al (16) in 2004, are multipotent stem cells that have been recently used in stem cell-mediated therapies and tissue engineering $(17,18)$. Studies have shown that hPDLSCs have an osteogenic potential both in vitro and in vivo, but the molecular mechanisms that underlie hPDLSC differentiation toward an osteoblastic phenotype remain elusive (19).

Thus, we hypothesized that ERR $\alpha$ may be expressed in PDLSCs and may be involved in the osteogenic characteristic of PDLSCs. In the present study, we found that $\operatorname{ERR} \alpha$ was expressed in hPDLSCs. Moreover, the expression level of ERR $\alpha$ was increased during the late period of osteogenic differentiation of hPDLSC. To confirm the role of ERR $\alpha$ in 
osteogenic differentiation of hPDLSCs, we used lentiviral delivery of miRNA to knock down the expression of ERR $\alpha$ in hPDLSCs, and found that the osteogenic potential of hPDLSCs was impaired after ERR $\alpha$ silencing. Our data indicate that ERR $\alpha$ may play an important role in osteogenic differentiation of hPDLSCs and may be used to improve the osteogenic potential of hPDLSCs. Thus, hPDLSCs may be a promising therapeutic target for the treatment of some bone diseases.

\section{Materials and methods}

Cell culture. Periodontal ligament (PDL) tissues were harvested from healthy premolars extracted for orthodontic reasons. Seven donors (12-16 years of age; four females and three males) and their parents provided informed consent. Approval was granted by the Ethics Committee of the School of Stomatology at the Fourth Military Medical University, China. PDL tissue attached to the middle third of the root was removed and cultured in phenol red-free $\alpha$-MEM supplemented with $10 \%$ charcoal-treated FBS (both from Gibco-BRL, Rockville, MD, USA), 2 mmol/1 glutamine, $100 \mathrm{U} / \mathrm{ml}$ penicillin and $100 \mu \mathrm{g} / \mathrm{ml}$ streptomycin at $37^{\circ} \mathrm{C}$ in a humidified atmosphere with $5 \% \mathrm{CO}_{2}$. After reaching $100 \%$ confluence, the cells were subcultured. To obtain homogeneous populations of PDLSCs, the limiting dilution technique was used as described previously (20). After 2-3 weeks in culture, single cell-derived clones were harvested and combined. Multiple colony-derived PDLSCs at passage 3 or 4 were used in experiments. The human breast adenocarcinoma cell line MCF-7 was cultured in the same manner and served as a positive control.

Flow cytometric analysis. The isolated putative hPDLSCs were collected and washed with PBS. To identify the PDLSC phenotype, $\sim 3 \times 10^{6}$ hPDLSCs were incubated with Alexa Fluor-conjugated monoclonal antibodies against human STRO-1 (340104), CD29 (303016), CD34 (343518), CD45 (304019) and CD105 (323209) (all from BioLegend, San Diego, CA, USA) for $2 \mathrm{~h}$ on ice. After washing twice and resuspending in PBS, the cells were analyzed using an Epics XL (Beckman Coulter, Fullerton, CA, USA).

Adipogenic induction. Adipogenic induction medium consisted of $\alpha$-MEM supplemented with $10 \%$ FBS, $2 \mu \mathrm{mol} / 1$ insulin (I6279), $0.5 \mathrm{mmol} / 1$ isobutyl-methylxanthine (I5879) and $10 \mathrm{nmol} / \mathrm{l}$ dexamethasone (D1756) (all from Sigma-Aldrich, St. Louis, MO, USA). PDLSCs were incubated in adipogenic induction medium for 14 days. The medium was replaced every other day. Intracellular lipid accumulation was detected by staining with Oil Red O (O0625; Sigma-Aldrich).

Osteogenic induction. Osteogenic induction medium consisted of $\alpha$-MEM supplemented with $10 \%$ FBS, $10 \mathrm{mmol} / \mathrm{l}$ $\beta$-glycerophosphate (G6251), $10 \mathrm{nmol} / 1$ dexamethasone, and $50 \mu \mathrm{g} / \mathrm{ml}$ ascorbic acid (A5960) (both from Sigma-Aldrich). PDLSCs were incubated in osteogenic induction medium for 21 days. The medium was replaced every other day. For ALP staining, cells were fixed and stained using a BCIP/NBT
Alkaline Phosphatase Color Development Kit (Beyotime, Haimen, China). Calcium accumulation was detected by staining with 2\% Alizarin Red S (pH 8.3, A5533; SigmaAldrich), and then rinsing extensively with PBS. After images of the nodules were recorded, $10 \%(\mathrm{w} / \mathrm{v})$ cetylpyridinium chloride (Sigma-Aldrich) was used to dissolve the nodules, and the absorbance was examined at $562 \mathrm{~nm}$.

RNA extraction and RT-PCR. Total RNA from PDLSCs was isolated using TRIzol reagent (Invitrogen, Carlsbad, CA, USA), according to the manufacturer's instructions. cDNA was synthesized from mRNA using M-MLV reverse transcriptase (Invitrogen). Primer sequences for ERR $\alpha$ and $\beta$-actin are shown in Table I. After predenaturation at $94^{\circ} \mathrm{C}$ for $5 \mathrm{~min}, 30 \mathrm{PCR}$ cycles were performed $\left(94^{\circ} \mathrm{C}\right.$ for $30 \mathrm{sec} ; 57^{\circ} \mathrm{C}$ for $30 \mathrm{sec}$; and $72^{\circ} \mathrm{C}$ for $30 \mathrm{sec}$ ), followed by a final extension at $72^{\circ} \mathrm{C}$ for $10 \mathrm{~min}$. PCR products were separated on $1.5 \%$ agarose gels containing ethidium bromide by electrophoresis and then visualized by a UV transilluminator.

Immunocytochemical analysis. PDLSCs and MCF-7 cells (positive control) were seeded on coverslips at a density of $5 \times 10^{3}$ cells $/ \mathrm{ml}$ for $48 \mathrm{~h}$, and then fixed with cold acetone. Immunocytochemical analysis was performed using the streptavidin-biotin complex method according to the manufacturer's protocol (Zhongshan Golden Bridge Biotechnology Co., Ltd., Beijing, China). DAB was used as the chromogen. The primary antibody against ERR $\alpha$ was a monoclonal rabbit anti-human ERR $\alpha$ (ab41868; Abcam, Cambridge, UK) at a 1:100 dilution. For the negative control, the primary antibody was substituted with a commensurable volume of PBS. The samples were counterstained with hematoxylin and examined under an Olympus compound microscope (Olympus, Tokyo, Japan) equipped with a Nikon digital camera (Nikon, Tokyo, Japan).

Viral vector construction and transduction. The BLOCK-iT ${ }^{\mathrm{TM}}$ RNAi Designer Program (Invitrogen) was used to design the miRNA sequence targeting the human ERR $\alpha$ gene. The target sequence was GCTACCCTCTGTGACCTCTTT. The annealed DNA sequences were cloned into pcDNA6.2-GW/EmGFP-miR (Invitrogen). The lentiviral vector plasmids were derived from the pLenti6.3/V5-Dest construct using the BLOCK-iT Lentiviral Pol II miR RNAi Expression System with EmGFP (Invitrogen), according to the manufacturer's instructions. Briefly, pcDNA6.2-GW/EmGFP-ERR $\alpha$-miR was recombined into the pLenti6.3/V5-Dest vector. The reaction mixtures were transformed into DH5a competent cells to select for positive clones. Sequencing was performed to verify the recombinant pLenti-ERR $\alpha$-miR plasmid. Lentiviruses were produced by transient transfection of 293FT cells using Lipofectamine 2000, lentiviral vectors, and packaging mix (Invitrogen). Transfection of hPDLSCs was performed by exposure to viral supernatant at a MOI of 100 in the presence of Polybrene $(8 \mathrm{mg} / \mathrm{ml}$; Sigma-Aldrich) for $48 \mathrm{~h}$. To produce stably transfected cell lines, the cells were cultured in selection medium containing $5 \mu \mathrm{g} / \mathrm{ml}$ blasticidin (Invitrogen) for 2 weeks. The cells were cultured in $\alpha$-MEM supplemented with $10 \%$ FBS and $2.5 \mu \mathrm{g} / \mathrm{ml}$ blasticidin to maintain and expand the cells. 
Table I. Primer sequences and product sizes for RT-PCR and quantitative real-time PCR.

\begin{tabular}{|c|c|c|c|}
\hline Target gene & $\begin{array}{c}\text { GeneBank } \\
\text { accession no. }\end{array}$ & Primer sequences & $\begin{array}{l}\text { Size of amplified } \\
\text { product (bp) }\end{array}$ \\
\hline $\mathrm{ERR} \alpha$ & NM_004451 & $\begin{array}{l}\text { F: GTGGGCGGCAGAAGTACAAG } \\
\text { R: GGTCAAAGAGGTCACAGAGGGT }\end{array}$ & 234 \\
\hline $\mathrm{OCN}$ & NM_199173 & $\begin{array}{l}\text { F: AGGGCAGCGAGGTAGTGAA } \\
\text { R: TCCTGAAAGCCGATGTGGT }\end{array}$ & 151 \\
\hline OPN & NM_000582 & $\begin{array}{l}\text { F: CTGATGCTACAGACGAGGACAT } \\
\text { R: GCTGTGGGTTTCAGCACTCT }\end{array}$ & 173 \\
\hline ALP & NM_000478 & $\begin{array}{l}\text { F: AGAACCCCAAAGGCTTCTTC } \\
\text { R: CTTGGCTTTTCCTTCATGGT }\end{array}$ & 74 \\
\hline RUNX2 & NM_004348 & $\begin{array}{l}\text { F: TCTACTATGGCACTTCGTCAGG } \\
\text { R: GCTTCCATCAGCGTCAACAC }\end{array}$ & 164 \\
\hline$\beta$-actin & NM_001101 & $\begin{array}{l}\text { F: TCCTTCCTGGGCATGGAGT } \\
\text { R: CAGGAGGAGCAATGATCTTGAT }\end{array}$ & 208 \\
\hline
\end{tabular}

F, forward; R, reverse.

Quantitative real-time PCR analysis. PDLSCs were treated with osteogenic induction medium, or cultured in standard medium as the control group. The cells were harvested at day 1, 5, 10, 15 and 20. Lentivirus-transduced PDLSCs were harvested after infection for $48 \mathrm{~h}$ to determine the efficiency of ERR $\alpha$ gene knockdown. After the production of stably transfected cell lines, osteogenic induction was performed, and the cells were harvested at day 7,14 and 21. Total RNA isolation and first-strand cDNA synthesis were performed as described above. Real-time PCR was carried out with a Mastercycler ep Realplex4 (Eppendorf AG, Hamburg, Germany) and SYBR-Green (Invitrogen). Primer sequences are shown in Table I. Reactions were performed under the following cycling conditions: $95^{\circ} \mathrm{C}$ for $10 \mathrm{~min}$, followed by 45 cycles of $95^{\circ} \mathrm{C}$ for $15 \mathrm{sec}$ and $60^{\circ} \mathrm{C}$ for $1 \mathrm{~min}$. Expression of the target genes was calculated using the formula $2^{-\Delta \Delta \mathrm{Ct}}$. Expression data were normalized to the expression of the $\beta$-actin gene.

Western blot analysis. Western blot analysis was used to confirm gene silencing as described previously (21). Briefly, cell extracts containing $30 \mu \mathrm{g}$ total protein were subjected to SDS-PAGE and then transferred onto PVDF membranes. The membranes were blocked and probed with primary antibodies that recognized ERR $\alpha$ (ab41868; Abcam) or $\beta$-actin (sc-47778; Santa Cruz Biotechnology Inc., Santa Cruz, CA, USA). Secondary antibodies were chosen according to the species of origin of the primary antibodies. After the incubation, the luminescent signals were detected using an enhanced chemiluminescence kit (Pierce, Rockford, IL, USA).

Statistical analyses. All experiments were performed at least three times. Each value is expressed as the mean \pm SD. Comparisons between two groups were performed by the independent samples t-test. Differences among three or more groups were analyzed by one-way ANOVA, followed by Dunnett's test for significance. Data with a P-value of $<0.05$ was considered to represent a statistically significant difference.

\section{Results}

Characterization of hPDLSCs. Stem-like cells were successfully isolated from the human PDL tissue. To characterize the phenotypic stem cell markers of single colony-derived PDLSCs, the expression levels of STRO-1, CD29, CD34, CD45 and CD105 were analyzed by flow cytometry. We observed that the hPDLSC population showed a high percentage of cells expressing markers of MSCs: STRO-1, CD29 and CD105 (Fig. 1A). In contrast, the cells were negative for the hematopoietic lineage marker CD34 and the leukocyte common antigen CD45 (Fig. 1A). To evaluate the multipotency of PDLSCs, we performed Oil Red O staining after culturing in adipogenic induction medium for 14 days and Alizarin Red S staining after culturing in osteogenic induction medium for 21 days. The results showed that hPDLSCs had strong adipogenic and osteogenic differentiation capacities (Fig. 1B and C).

Expression of ERR $\alpha$ in hPDLSCs. Immunocytochemistry and RT-PCR analyses were employed to examine the expression of ERR $\alpha$ in cultured hPDLSCs. As shown in Fig. 2A, a clear band representing ERR $\alpha$ was detected in the hPDLSCs at the same molecular weight as that in positive control cells (MCF-7 cells). Immunocytochemical staining confirmed the positive expression of ERR $\alpha$ protein in the hPDLSCs (Fig. 2B) and MCF-7 cells (Fig. 2C). Positive signals in the nuclei were stronger than those in the cytoplasm of the hPDLSCs (Fig. 2B). In contrast, no positive signal was found in the negative control (Fig. 2D).

Temporal expression of ERR $\alpha$ in hPDLSCs treated with osteogenic induction medium. To investigate the role of ERR $\alpha$ 

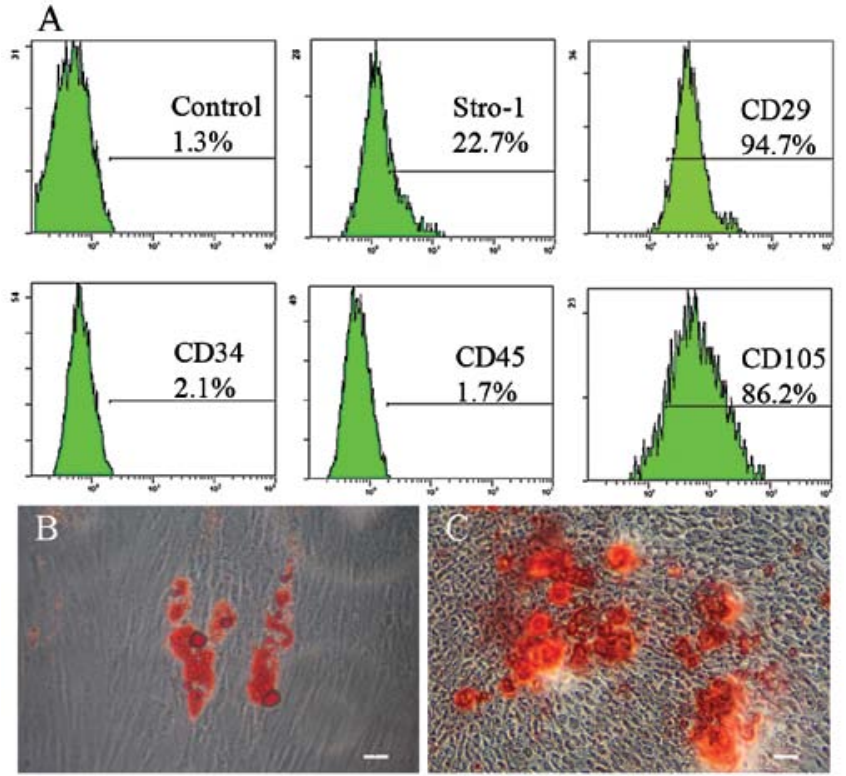

Figure 1. Characterization of hPDLSCs. (A) Flow cytometric analysis of hPDLSC biomarkers. Histograms show the percentage of cells expressing Stro-1, CD29, CD34, CD45 and CD105 in the hPDLSC population. (B) Adipogenic differentiation of hPDLSCs. Cells were cultured in adipogenic induction medium containing insulin, isobutyl-methylxanthine and dexamethasone for 14 days. Lipid droplets were stained with Oil Red O. (C) Osteogenic differentiation of hPDLSCs. Cells were cultured in osteogenic induction medium containing $\beta$-glycerophosphate, dexamethasone and ascorbic acid for 21 days. The mineralized nodules were stained with Alizarin Red S. Scale bar, $20 \mu \mathrm{m}$.
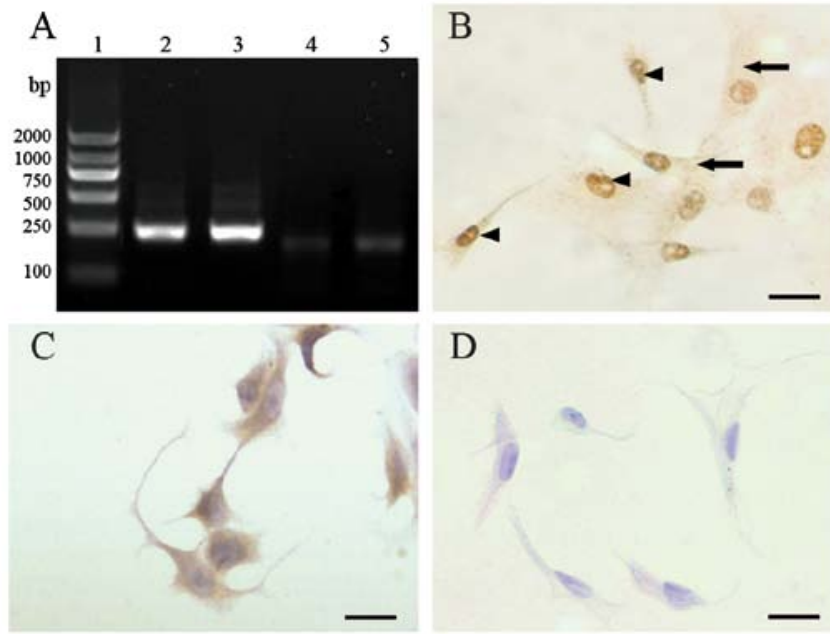

D

Figure 2. RT-PCR and immunocytochemical staining of ERR $\alpha$ in hPDLSCs and MCF-7 cells. (A) Analysis of RT-PCR products on agarose gels containing ethidium bromide and visualization under UV illumination. Lane 1, Marker; lane $2, \beta$-actin mRNA in hPDLSCs; lane 3, $\beta$-actin mRNA in MCF-7 cells; lane 4, ERR $\alpha$ mRNA in PDLSCs; lane 5. ERR $\alpha$ mRNA in MCF-7 cells. (B) Positive staining of ERR $\alpha$ in the cytoplasm (arrows) and nuclei (arrowheads) of hPDLSCs. (C) Positive staining for ERR $\alpha$ in MCF-7 cells. (D) Negative immunoreactivity for ERR $\alpha$ in hPDLSCs. Scale bar, $20 \mu \mathrm{m}$.

in osteogenic differentiation of hPDLSCs, we measured the expression of ERR $\alpha$ mRNA during osteogenic induction (day 1, 7, 14 and 21) of hPDLSCs by quantitative real-time PCR (Fig. 3). There were no significant differences between the

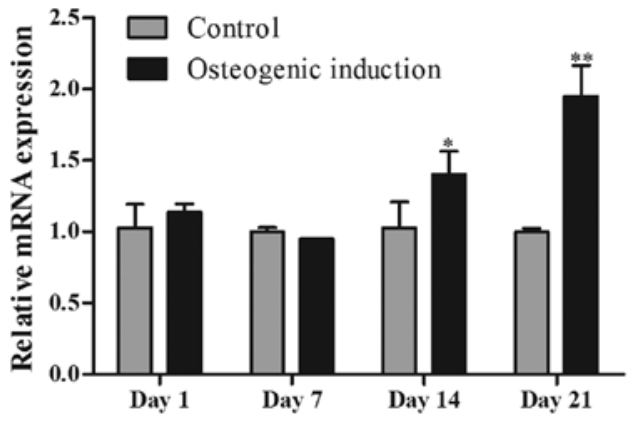

Figure 3. ERR $\alpha$ mRNA expression in osteogenic-induced hPDLSCs. Cells were collected at day $1,7,14$ and 21 . ERR $\alpha$ mRNA levels were determined by real-time PCR, and the values were normalized to $\beta$-actin mRNA expression. Relative mRNA levels were calculated by giving an arbitrary value of 1.0 to the untreated group. Each bar represents the mean $\pm \operatorname{SD}(n=3)$. ${ }^{*} \mathrm{P}<0.05,{ }^{* *} \mathrm{P}<0.01$ vs.the untreated group.

osteogenic induction group and the control group at day 1 and 7 . As the cells entered the mineralization stage after culturing for 14 days, ERR $\alpha$ mRNA expression in the osteogenic induction group was significantly increased $(\mathrm{P}<0.05)$, and at day 21 , it reached a peak level compared with that in the control group $(\mathrm{P}<0.001)$.

Knockdown of the expression of ERR $\alpha$ results in decreased osteogenic differentiation of hPDLSCs. To further analyze the biological function of ERR $\alpha$, we used lentiviral vectors driving the expression of miRNA against the ERR $\alpha$ gene. DNA sequencing results revealed that the inserted fragments were correct, and no mutations were found in the recombinant plasmids. Successful transfection of recombinant plasmids into hPDLSCs was confirmed by detecting EmGFP expression under a fluorescence microscope (Fig. 4A-D). After stably transfected cell lines were produced by culturing in selection medium containing blasticidin for 2 weeks, flow cytometric analysis showed that the transfection rate of LV-miR-neg and LV-miR-ERR $\alpha$ was $>90 \%$. Fig. $4 \mathrm{~F}-\mathrm{H}$ shows the EmGFP expression in hPDLSCs stably transfected with LV-miR-neg or LV-miR-ERR $\alpha$. Gene silencing was confirmed by real-time PCR after culturing in osteogenic induction medium for 7, 14 and 21 days, and by western blot analysis at day 7 (Fig. 4I and J). The results showed that transfection with LV-miR-ERR $\alpha$ decreased ERR $\alpha$ mRNA and protein expression by $\sim 50 \%$.

Next, we determined the osteogenic capacity of hPDLSCs transfected with LV-miR-ERR $\alpha$, and used the cells transfected with LV-miR-neg as the control. ALP and Alizarin Red S staining were performed to detect the mineralization of hPDLSCs (Fig. 5A). The density of ALP staining at day 14 was lower in the ERR $\alpha$-knockdown group than that in the control group $(\mathrm{P}<0.01)$ and Alizarin Red $\mathrm{S}$ staining at day 21 also showed a significant decrease in the mineralization of hPDLSCs transfected with LV-miR-ERR $\alpha(\mathrm{P}<0.01)$.

We next monitored the mRNA expression of some mineralization-related genes in hPDLSCs cultured in osteogenic induction medium at day 7,14 and 21. We observed significant decreases in ALP, RUNX2, OCN and OPN mRNA levels in ERR $\alpha$-knockdown cells $(\mathrm{P}<0.05)$ (Fig. 5B). Taken together, our data indicate that ERR $\alpha$ promotes osteogenic differentiation of hPDLSCs. 

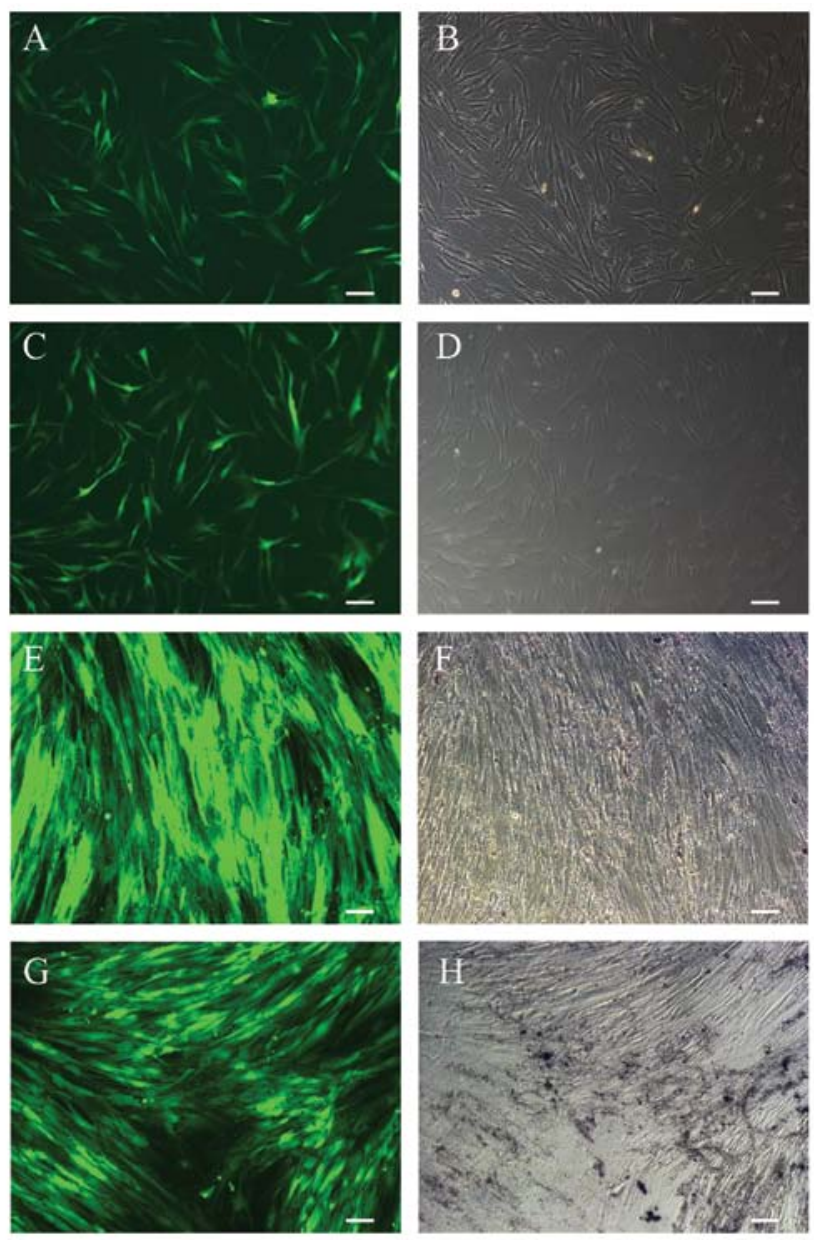

I

ERR $\alpha$

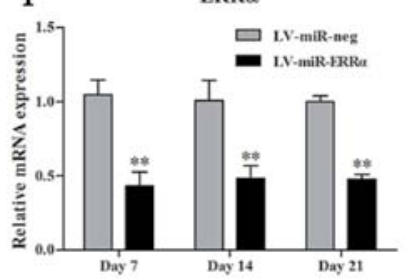

$\mathrm{J}$

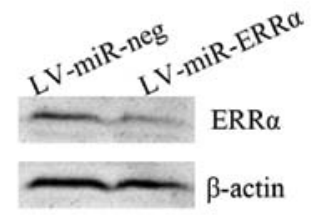

Figure 4. Gene transduction. (A and B) EmGFP expression in hPDLSCs at day 3 after transduction of LV-miR-neg. (C and D) EmGFP expression in hPDLSCs at day 3 after transduction of LV-miR-ERR $\alpha$. (E and F) hPDLSCs stably transfected with LV-miR-neg expressing EmGFP were cultured in osteogenic induction medium for 14 days. (G and $\mathrm{H}$ ) hPDLSCs stably transfected with LV-miR-ERR $\alpha$ expressing EmGFP were cultured in osteogenic induction medium for 14 days. Scale bar, $50 \mu \mathrm{m}$. (I) Quantitative real-time PCR analysis of ERR $\alpha$ mRNA expression in hPDLSCs transduced with LV-miR-neg or LV-miR-ERR $\alpha$. The values were normalized to $\beta$-actin mRNA expression. Relative mRNA levels were calculated by assigning an arbitrary value of 1.0 to LV-miR-neg cells. Data are presented as the mean $\pm \mathrm{SD}(\mathrm{n}=3) .{ }^{* *} \mathrm{P}<0.01$ vs. control group. (J) Western blot analysis of ERR $\alpha$ and $\beta$-actin protein expression at day 3 after gene transduction.

\section{Discussion}

ERR $\alpha$ is capable of regulating the transcription of genes involved in multiple cellular and physiological processes $(3,8,22)$. Studies have established the key roles of ERR $\alpha$ in regulating mitochondrial biogenesis (3), fatty acid oxidation (23) and oxidative phosphorylation (3) and have correlated ERR $\alpha$ with various types of cancer and metabolic disor- ders (22). ERR $\alpha$ has also been proposed as an important regulatory factor of bone metabolism (8) and is regarded as a potential therapeutic target for treating osteoporosis (24).

Previous studies have shown that ERR $\alpha$ is selectively expressed in a variety of cell types during development and in adult tissues, and ERR $\alpha$ expression increases according to metabolic demands $(8,23)$. ERR $\alpha$ is highly expressed during the formation of ossification zones during mouse development in vivo (25), as well as in primary rat calvarial cells in vitro (9). Nevertheless, its expression in periodontal tissues has not been studied. PDLSCs are isolated from the PDL that connects two types of hard tissues, tooth cementum and alveolar bone, and thus express an array of cementoblastic/osteoblastic markers. PDLSCs are capable of forming cementum/PDL-like tissue, and participate in the repair of alveolar bone (16). In addition, healthy cells can be easily obtained from adolescents who require teeth extraction for orthodontic reasons. Therefore, PDLSCs are ideal seed cells for periodontal tissue engineering therapies. Since PDL is also a highly metabolically active tissue with peculiar mechanical/functional demands (26), we investigated ERR $\alpha$ expression in PDLSCs in vitro.

Osteogenic differentiation of hPDLSCs is a complex process regulated by multiple signals at different levels. Since ERR $\alpha$ was expressed in hPDLSCs, we assumed that ERR $\alpha$ was also involved in osteogenic differentiation of hPDLSCs. We monitored the expression of ERR $\alpha$ as the cells were induced to differentiate in osteogenic induction medium, and found a gradual increase in the expression levels of ERR $\alpha$. We next used RNA interference to inhibit ERR $\alpha$ expression in hPDLSCs. Downregulation of ERR $\alpha$ significantly inhibited the mineralization capacity of hPDLSCs. We further detected the expression of mineralization-related genes during the osteogenic differentiation of hPDLSCs. ALP and OCN are regarded as indicators of early and late osteogenesis, respectively. RUNX2 is a key transcription factor essential for the commitment of multipotent mesenchymal cells to the osteoblast lineage, and serves as an early transcriptional regulator of osteogenic differentiation (27). OPN is a noncollagenous bone matrix protein, a marker of the late stages of osteoblastic differentiation, and its promoter can be transactivated by ERR $\alpha$ (28). These mineralization-related genes were downregulated by lentiviral-mediated ERR $\alpha$ knockdown, suggesting that ERR $\alpha$ initiates early-stage osteogenic differentiation and maintains late-stage osteogenic differentiation of hPDLSCs in vitro.

In a recent study, Rajalin et al (12) also proposed a positive role for ERR $\alpha$ in osteoblastic differentiation of MSCs using ERR $\alpha$-knockout (KO) mice. Auld et al (13) showed similar results regarding the role of ERR $\alpha$ in mineralization of human MSCs and they found that native ERR $\alpha$ represses Wnt signaling, which has been shown to suppress osteogenesis in the human MSC system (29). Our results also showed that lentiviral-mediated ERR $\alpha$ knockdown reduced the osteogenic differentiation of PDLSCs that are tissue-specific MSCs. However, the mechanism by which ERR $\alpha$ enhances the osteogenic potential of MSCs is still unclear. Previous studies have shown that overexpression of ERR $\alpha$ leads to induction of the p21 cell cycle inhibitor, and inhibition of proliferation in breast cell lines $(30,31)$. Based on these studies, we inferred that, upon silencing in MSCs, ERR $\alpha$ may enhance cell expan- 
A
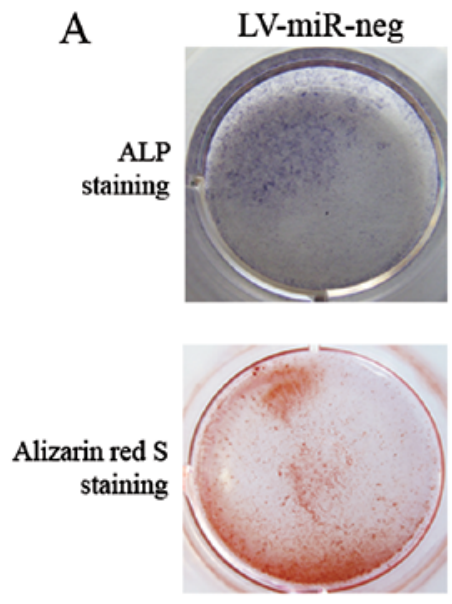

B
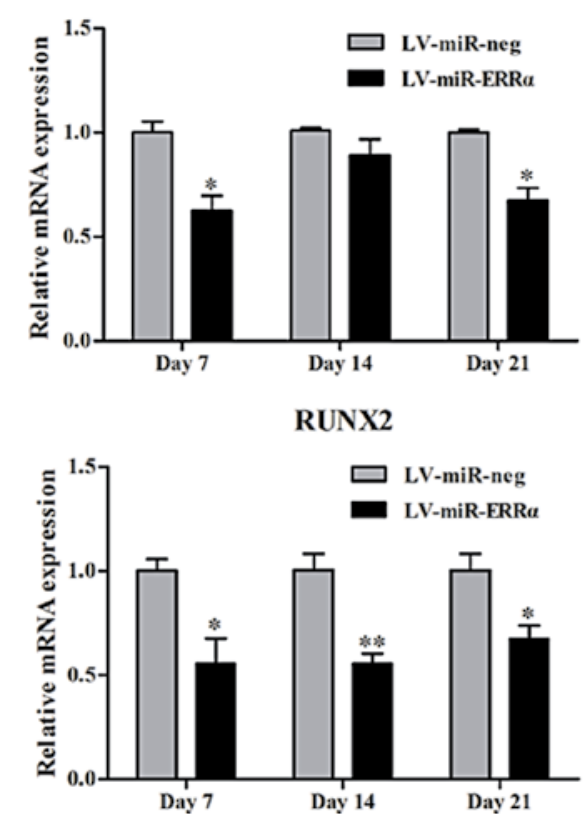

LV-miR-ERR $\alpha$
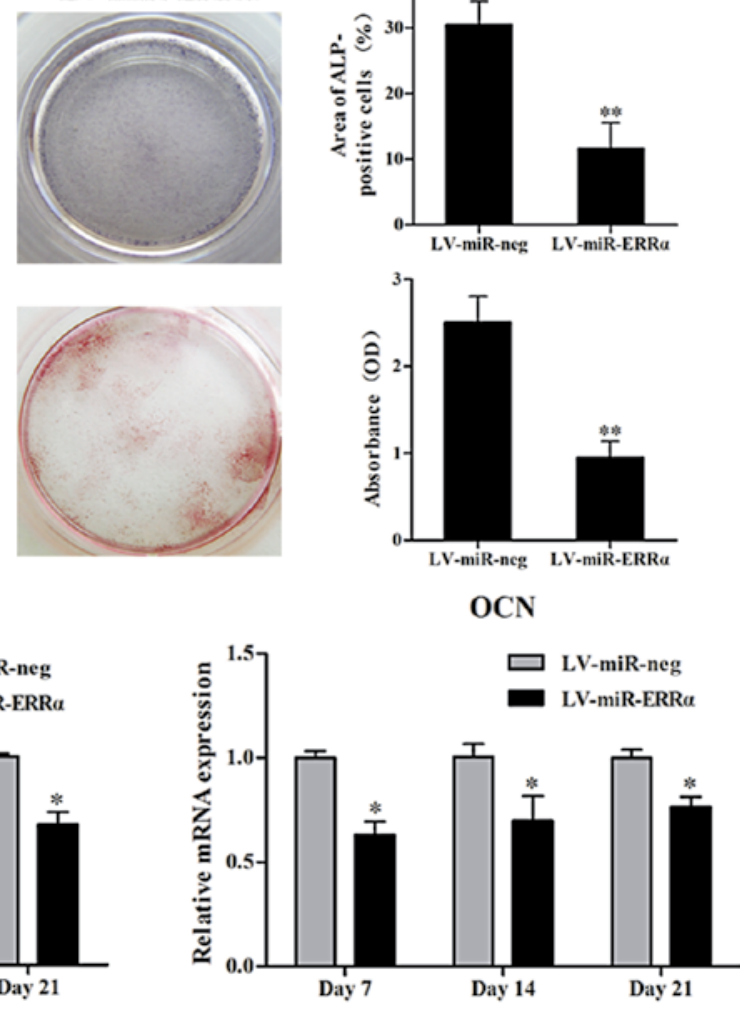

OPN

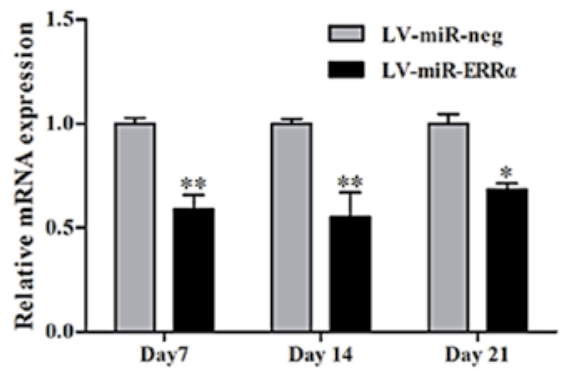

Figure 5. Effect of ERR $\alpha$ silencing on calcium deposition and osteoblastic marker expression during osteogenic differentiation of hPDLSCs. (A) ALP and Alizarin Red S staining were performed at day 7 and 14, respectively, after osteogenic induction. The graphs show the statistically significant differences in ALP-positive cells and the quantification of mineralization nodules between the two groups. (B) Quantitative real-time PCR analysis of ALP, OCN, RUNX2, and OPN mRNA expression in hPDLSCs treated with osteoblastic induction medium for 7, 14 and 21 days. The values were normalized to $\beta$-actin mRNA expression. Relative mRNA levels were calculated by assigning an arbitrary value of 1.0 to LV-miR-neg cells. Data are presented as means \pm SD ( $=3$ ). ${ }^{*} \mathrm{P}<0.05,{ }^{* *} \mathrm{P}<0.01$ vs. the control group.

sion and lead to delayed differentiation. However, a report by Delhon et al (14) showed a negative effect of ERR $\alpha$ on bone formation both in vivo and in vitro. Teyssier et al (15) also found that ERR $\alpha$ negatively regulates osteogenic differentiation in vitro and demonstrated a gender-dependent effect of $\mathrm{ERR} \alpha$ in ERR $\alpha-\mathrm{KO}$ mice. These contradictions may result from differences in the tissues and species, variability of the primary cell cultures, differences in the osteogenic culture conditions, the genetic backgrounds of the ERR $\alpha$-KO mice, or potential gender-dependent effects of ERR $\alpha$ (12).

ERR $\alpha$ has crosstalk with ERs and estrogen and modulates ER-mediated signaling pathways (8). Studies have shown that $\mathrm{ERR} \alpha$ is a potential therapeutic target of postmenopausal osteoporosis (24). Since postmenopausal osteoporosis is a well-known systemic inflammatory environment, and periodontitis is also a chronic inflammatory microenviron- ment, we theorized that ERR $\alpha$ may be involved in periodontal disease (32). Previous studies by our group demonstrated that hPDLSCs derived from patients with chronic periodontitis (P-PDLSCs) display an impaired osteogenic potential compared with that of hPDLSCs derived from healthy donors (H-PDLSCs) (33). We also observed that ERR $\alpha$ expression is decreased in P-PDLSCs, and when H-PDLSCs are treated with TNF- $\alpha$, the main proinflammatory factor of periodontitis, the expression of ERR $\alpha$ is significantly decreased along with the attenuation of osteogenic differentiation (unpublished data). Similarly, Bonnelye et al (34) found that ERR $\alpha$ mRNA expression is downregulated in the subchondral bone of mice with induced joint inflammation, which is paralleled by downregulation of markers of bone formation. Therefore, ERR $\alpha$ may be involved in impaired osteogenic differentiation of hPDLSCs in periodontal disease, and we believe that 
ERR $\alpha$ may also be a promising therapeutic target for treating inflammatory bone diseases such as local periodontitis and systemic osteoporosis. However, the pathway through which inflammatory diseases affect ERR $\alpha$ expression and the relationship between ERR $\alpha$, ERs and estrogen are far from clear and need further investigation.

In conclusion, we detected the expression of ERR $\alpha$ in hPDLSCs in vitro and observed positive effects of ERR $\alpha$ on the osteogenic differentiation of hPDLSCs. This result suggests that ERR $\alpha$ regulates osteogenic differentiation of PDLs and may be involved in the pathogenesis of estrogenrelated periodontal disease. Further studies are required to investigate the specific functions of $E R R \alpha$ in periodontal tissues including periodontal ligaments, gingiva and alveolar bone under different physiological and pathophysiological conditions, and the crosstalk between ERRs and ERs to elucidate the mechanisms through which ERR $\alpha$ acts both in bone loss due to estrogen deficiency and in periodontal issues.

\section{Acknowledgements}

This study was supported by the Nature Science Foundation of China (grant 30872913). We thank Dr Jiaxing Zhou for generously providing the MCF-7 cell line. We also acknowledge Professor Hui $\mathrm{Xu}$ for critical reading of the manuscript.

\section{References}

1. Giguère V, Yang N, Segui $P$ and Evans RM: Identification of a new class of steroid hormone receptors. Nature 331: 91-94, 1988.

2. Vanacker JM, Bonnelye E, Chopin-Delannoy S, Delmarre C, Cavaillès $\mathrm{V}$ and Laudet V: Transcriptional activities of the orphan nuclear receptor ERR $\alpha$ (estrogen receptor-related receptor- $\alpha$ ). Mol Endocrinol 13: 764-773, 1999.

3. Huss JM, Torra IP, Staels B, Giguere V and Kelly DP: Estrogen-related receptor $\alpha$ directs peroxisome proliferatoractivated receptor $\alpha$ signaling in the transcriptional control of energy metabolism in cardiac and skeletal muscle. Mol Cell Biol 24: 9079-9091, 2004

4. Luo J, Sladek R, Carrier J, Bader JA, Richard D and Giguère V: Reduced fat mass in mice lacking orphan nuclear receptor estrogen-related receptor $\alpha$. Mol Cell Biol 23: 7947-7956, 2003.

5. Villena JA, Hock MB, Chang WY, Barcas JE, Giguère V and Kralli A: Orphan nuclear receptor estrogen-related receptor $\alpha$ is essential for adaptive thermogenesis. Proc Natl Acad Sci USA 104: 1418-1423, 2007.

6. Fujimoto J and Sato E: Clinical implication of estrogen-related receptor (ERR) expression in uterine endometrial cancers. J Steroid Biochem Mol Biol 116: 71-75, 2009.

7. Lu D, Kiriyama Y, Lee KY and Giguère V: Transcriptional regulation of the estrogen-inducible pS2 breast cancer marker gene by the ERR family of orphan nuclear receptors. Cancer Res 61: 6755-6761, 2001

8. Bonnelye E and Aubin JE: Estrogen receptor-related receptor $\alpha$ : a mediator of estrogen response in bone. J Clin Endocrinol Metab 90: 3115-3121, 2005

9. Bonnelye E, Merdad L, Kung V and Aubin JE: The orphan nuclear estrogen receptor-related receptor $\alpha(E R R \alpha)$ is expressed throughout osteoblast differentiation and regulates bone formation in vitro. J Cell Biol 153: 971-984, 2001.

10. Bonnelye E and Aubin JE: Differential expression of estrogen receptor-related receptor $\alpha$ and estrogen receptors $\alpha$ and beta in osteoblasts in vivo and in vitro. J Bone Miner Res 17: 1392-1400, 2002.

11. Bonnelye E, Saltel F, Chabadel A, Zirngibl RA, Aubin JE and Jurdic P: Involvement of the orphan nuclear estrogen receptorrelated receptor $\alpha$ in osteoclast adhesion and transmigration. J Mol Endocrinol 45: 365-377, 2010.
12. Rajalin AM, Pollock $\mathrm{H}$ and Aarnisalo P: ERR $\alpha$ regulates osteoblastic and adipogenic differentiation of mouse bone marrow mesenchymal stem cells. Biochem Biophys Res Commun 396: 477-482, 2010

13. Auld KL, Berasi SP, Liu Y, et al: Estrogen-related receptor $\alpha$ regulates osteoblast differentiation via $\mathrm{Wnt} / \beta$-catenin signaling. J Mol Endocrinol 48: 177-191, 2012.

14. Delhon I, Gutzwiller S, Morvan F, et al: Absence of estrogen receptor-related- $\alpha$ increases osteoblastic differentiation and cancellous bone mineral density. Endocrinology 150: 4463-4472, 2009.

15. Teyssier C, Gallet M, Rabier B, et al: Absence of ERR $\alpha$ in female mice confers resistance to bone loss induced by age or estrogen-deficiency. PLoS One 4: e7942, 2009.

16. Seo BM, Miura M, Gronthos S, et al: Investigation of multipotent postnatal stem cells from human periodontal ligament. Lancet 364: 149-155, 2004.

17. Trubiani O, Orsini G, Zini N, et al: Regenerative potential of human periodontal ligament derived stem cells on threedimensional biomaterials: a morphological report. J Biomed Mater Res A 87: 986-993, 2008.

18. Liu Y, Zheng Y, Ding G, et al: Periodontal ligament stem cellmediated treatment for periodontitis in miniature swine. Stem Cells 26: 1065-1073, 2008.

19. Chadipiralla K, Yochim JM, Bahuleyan B, et al: Osteogenic differentiation of stem cells derived from human periodontal ligaments and pulp of human exfoliated deciduous teeth. Cell Tissue Res 340: 323-333, 2010.

20. Pan F, Zhang R, Wang G and Ding Y: Oestrogen receptors are involved in the osteogenic differentiation of periodontal ligament stem cells. Biosci Rep 31: 117-124, 2011.

21. Liang L, Yu JF, Wang Y, Wang G and Ding Y: Effect of estrogen receptor beta on the osteoblastic differentiation function of human periodontal ligament cells. Arch Oral Biol 53: 553-557, 2008.

22. Ariazi EA and Jordan VC: Estrogen-related receptors as emerging targets in cancer and metabolic disorders. Curr Top Med Chem 6: 203-215, 2006.

23. Sladek R, Bader JA and Giguère V: The orphan nuclear receptor estrogen-related receptor $\alpha$ is a transcriptional regulator of the human medium-chain acyl coenzyme A dehydrogenase gene. Mol Cell Biol 17: 5400-5409, 1997.

24. Gallet M and Vanacker JM: ERR receptors as potential targets in osteoporosis. Trends Endocrinol Metab 21: 637-641, 2010.

25. Bonnelye E, Vanacker JM, Dittmar T, et al: The ERR-1 orphan receptor is a transcriptional activator expressed during bone development. Mol Endocrinol 11: 905-916, 1997.

26. Hassell TM, Rateitschak KH, Wolf HF and RateitschakPluss EM: Color Atlas of Dental Medicine: Periodontology. Wolf HF and Rateitschak KH (eds.). Thieme, Stuttgart, 2005.

27. Komori T: Regulation of bone development and extracellular matrix protein genes by RUNX2. Cell Tissue Res 339: 189-195, 2010.

28. Vanacker JM, Delmarre C, Guo X and Laudet V: Activation of the osteopontin promoter by the orphan nuclear receptor estrogen receptor related $\alpha$. Cell Growth Differ 9: 1007-1014, 1998.

29. Liu G, Vijayakumar S, Grumolato L, et al: Canonical Wnts function as potent regulators of osteogenesis by human mesenchymal stem cells. J Cell Biol 185: 67-75, 2009.

30. Bianco S, Lanvin O, Tribollet V, Macari C, North S and Vanacker JM: Modulating estrogen receptor-related receptor- $\alpha$ activity inhibits cell proliferation. J Biol Chem 284: 23286-23292, 2009.

31. Castet A, Herledan A, Bonnet S, Jalaguier S, Vanacker JM and Cavaillès V: Receptor-interacting protein 140 differentially regulates estrogen receptor-related receptor transactivation depending on target genes. Mol Endocrinol 20: 1035-1047, 2006.

32. Geurs NC: Osteoporosis and periodontal disease. Periodontol 2000 44: 29-43, 2007.

33. Liu Y, Liu W, Hu C, et al: MiR-17 modulates osteogenic differentiation through a coherent feed-forward loop in mesenchymal stem cells isolated from periodontal ligaments of patients with periodontitis. Stem Cells 29: 1804-1816, 2011.

34. Bonnelye E, Laurin N, Jurdic P, Hart DA and Aubin JE: Estrogen receptor-related receptor- $\alpha($ ERR- $\alpha)$ is dysregulated in inflammatory arthritis. Rheumatology 47: 1785-1791, 2008. 